\title{
MicroRNA-497-5p negatively regulates the proliferation and cisplatin resistance of non-small cell lung cancer cells by targeting YAP1 and TEAD1
}

\author{
Shang-Gan Zeng ${ }^{1}$, Jian-Hong Xie ${ }^{2}$, Qun-Ying Zeng ${ }^{2}$, Shao-Hua Dai ${ }^{1}$, Yun Wang ${ }^{1}$, Xue-Mei Wan ${ }^{3}$, \\ Xue-Liang Zhou ${ }^{3}$
}

${ }^{1}$ Department of Thoracic Surgery, The First Affiliated Hospital of Nanchang University, Nanchang 330006, China; ${ }^{2}$ Department of Surgery, Suichuan People's Hospital, Ji'an 343900, China; ${ }^{3}$ Department of Cardiovascular Surgery, The First Affiliated Hospital of Nanchang University, Nanchang 330006, China

Contributions: (I) Conception and design: JH Xie, QY Zeng; (II) Administrative support: SG Zeng; (III) Provision of study materials or patients: XM Wan, XL Zhou; (IV) Collection and assembly of data: Y Wang; (V) Data analysis and interpretation: QY Zeng, SH Dai; (VI) Manuscript writing: All authors; (VII) Final approval of manuscript: All authors.

Correspondence to: Dr. Xue-Liang Zhou. Department of Cardiovascular Surgery, The First Affiliated Hospital of Nanchang University, No.17, Yong Wai Zheng Street, Nanchang 330006, China. Email: zhouxueliang09@163.com.

Background: MicroRNAs (miRNAs) are crucial regulators in the pathological processes and drug resistance of lung cancer. In this study, we investigated the role of miR-497-5p in modulating the function of non-small cell lung cancer (NSCLC).

Methods: MiR-497-5p expression in lung cancer tissues and cells was evaluated by qRT-PCR. Cell proliferation was evaluated by CCK-8 assay and colony-formation assay. Cell cycle and cell apoptosis were detected by flow cytometry. The effect of miR-497-5p on the expression of Yes-associated protein 1 (YAP1) and TEA domain family member 1 (TEAD1) was analyzed by qRT-PCR, Western blot and luciferase activity assay.

Results: The expression of miR-497-5p was significantly downregulated in lung cancer tissues and cells compared with paired normal tissues and cells. Overexpression of miR-497-5p induced growth retardation and apoptosis of A549 lung cancer cells. Mechanistically, YAP1 and TEAD1 were targeted and downregulated by miR-497-5p. Finally, we found that miR-497-5p increased cisplatin chemosensitivity in A549 cells.

Conclusions: MiR-497-5p suppresses cell proliferation and resistance to cisplatin in NSCLC by downregulating the expression of YAP1 and TEAD1.

Keywords: Cisplatin resistance; miR-497-5p; proliferation; TEA domain family member 1 (TEAD1); Yesassociated protein 1 (YAP1)

Submitted Mar 26, 2019. Accepted for publication Aug 27, 2019.

doi: $10.21037 /$ tcr.2019.10.03

View this article at: http://dx.doi.org/10.21037/tcr.2019.10.03

\section{Introduction}

Lung cancer is the most common malignancy worldwide with high prevalence and high mortality. It is estimated that the 5 -year overall survival rate of lung cancer is less than $15 \%$ (1). Lung cancer can generally be classified into the two major types based on the histological features, small cell lung cancer (SCLC) and non-small cell lung cancer (NSCLC) (2). Although several drugs targeting key oncogenic molecules have been applied in the clinical treatment of NSCLC, such epidermal growth factor 
receptor (EGFR) inhibitors Gefitinib and Afatinib, or anaplastic lymphoma kinase (ALK) inhibitors crizotinib and ceritinib, these drugs are only effective in patients harboring mutations in corresponding genes $(3,4)$. In terms of chemotherapy, continuous or repeated administration of chemotherapeutic drugs often induces chemoresistance in NSCLC cells, leading to the final failure of chemotherapy $(5,6)$. Identifying novel molecular regulators in NSCLC initiation or progression maybe helpful for understanding the pathological processes of NSCLC, and allow for the development of relevant therapeutic strategies.

MicroRNAs are non-coding, short RNA molecules which can interfere the expression of their target genes either by degrading their mRNA or inhibiting translation $(7,8)$. The abnormal expression of miRNAs was observed in diverse kinds of human cancers, including NSCLC (9-12). A collection of these dysregulated miRNAs has been identified as biomarkers in NSCLC to facilitate the diagnosis or sub-classification of NSCLC, such as miR1254, miR-574-5p, miR-1253, miR-504, and miR-26a-5p (13-15). Apart from serving as biomarkers, miRNAs are crucial regulators in lung cancer pathology. For example, targeting miR-224, miR-211 was shown to inhibit the growth of NSCLC $(16,17)$. On the other hand, inhibition of miRNA-328 decreased cisplatin resistance in NSCLC (18). These studies suggest that miRNAs can serve as candidate therapeutic targets for NSCLC (9).

MiR-497-5p was reported to function as a tumor suppressor in some human cancers. For instance, miR-497-5p inhibits the proliferation and invasion of angiosarcoma cells through downregulating by targeting $\mathrm{KCa} 3.1$, an oncogenic potassium channel protein (19). Also, miR-497-5p reduces multiple malignant behaviors of melanoma cells (20). Besides, miR-497 increases the resistance to temozolomide in human glioma cells (21). However, whether miR-497 can regulate the growth or chemoresistance of NSCLC remains unknown.

In the present study, we reported that miR-497-5p attenuated the proliferation and increased the apoptosis of A549 lung cancer cells. Mechanistically, miR-497-5p targets and downregulates the expression of Yes-associated protein 1 (YAP1) and TEA domain family member 1 (TEAD1) in A549 cells. In addition, miR-497-5p was found to sensitize A549 cells to cisplatin treatment. Thus, our study identifies miR-497-5p served as an antitumor miRNA and represents a novel therapeutic target for NSCLC patients with cisplatin resistance.

\section{Methods}

\section{Clinical specimens}

The specimens in this study were obtained from 32 patients who were all pathologically diagnosed with NSCLC at The First Affiliated Hospital of Nanchang University. Specimens were collected at surgery, and were frozen immediately in liquid nitrogen then stored at $-80{ }^{\circ} \mathrm{C}$ for RNA extraction. The study has been approved by the Hospital's Ethics Committee (Approval Number: CDYFY2017-8999). Inform consent was obtained from all patients.

\section{Cell culture}

A549, BEAS-2B, H1299, HCC-827, NCI-H358 cell lines were obtained from (American Type Culture Collection, ATCC) and were cultured as recommended by the supplier. A549/DDP cells were obtained from Shanghai YiYan Biotechnology Company (China). A549 cells and A549/DDP cells were cultured in RPMI 1640 medium supplemented with $10 \%$ fetal bovine serum (Biowest, Spain) in a humidified atmosphere with $5 \% \mathrm{CO}_{2}$ at $37^{\circ} \mathrm{C}$.

\section{Cell transfection}

The human miR-497-5p mimics/inhibitor and their corresponding miRNA controls were synthesized by GenePharma (Shanghai, China). YAP1 and TEAD1 expression pcDNA3.1 plasmids were purchased from Origene (Beijing, China). Lipofectamine 2000 Reagent (Invitrogen, Carlsbad, CA, USA) was used for cell transfection according to the manufacturer's instructions.

\section{CCK-8 assay}

$8 \times 10^{4}$ A549 cells were seeded into 96-well plates and cultured overnight. CCK- 8 assay was performed using CCK-8 Kit (Beyotime, China) according to the manufacturer's instruction. Briefly, 10× CCK-8 solution was added into each well. Two hours later, the optical density values (OD values) were measured on a microplate reader (Thermo Fisher Scientific) at $490 \mathrm{~nm}$.

\section{Colony formation assay}

A549 cells were seeded in 6-well plates and were cultured for 10 days. Cell colonies were fixed in $70 \%$ ethanol for 
Table 1 Primer sequences for quantitative real-time PCR

\begin{tabular}{lll}
\hline Gene & Forward sequence & Reverse sequence \\
\hline MiR-497-5p & 5'-GCGCAGCAGCACACTGTG-3' & 5'-GTGCAGGGTCCGAGGT-3' \\
YAP1 & 5'-TAGCCCTGCGTAGCCAGTTA-3' & 5'-TCATGCTTAGTCCACTGTCTGT-3' \\
TEAD1 & 5'-CCCTGGCTATCTATCCACCA-3' & 5'-AGGGCCTTATCCTTTGCAGT-3' \\
$\beta$-Actin & 5'-CATGTACGTTGCTATC-3' & 5'-CTCCTTAATGTCACGCACGAT-3' \\
U6 & 5'-CTCGCTTCGGCAGCACA-3' & 5'-AACGCTTCACGAATTTGCGT-3' \\
\hline
\end{tabular}

2 hours and stained with $0.1 \%$ crystal violet for 5 minutes (Beyotime, China). Colony formation was photographed and counted under a light microscope.

\section{Cell cycle assay}

A549 cells in 6-well plates transfected with different combinations of miR-497-5p mimic, miR-497-5p inhibitor, YAP1 or TEAD1 were dissociated with trypsin and were fixed in $70 \%$ ethanol for 12 hours, followed by PBS washes. Then $5 \mu \mathrm{L}$ of PI was added to each tube. Cell cycle was analyzed by flow cytometry using on a NovoCyte Flow Cytometers (ACEA, USA).

\section{Cell apoptosis assay}

A549 cells in 6-well plates transfected with different combinations of miR-497-5p mimic, miR-497-5p inhibitor, YAP1 or TEAD1 were dissociated with trypsin and were stained with FITC-annexin V (Biolegend, USA) and propidium iodide (PI) (Biolegend, USA) for $20 \mathrm{~min}$ in dark at room temperature. Cell apoptosis was analyzed by flow cytometry using on a NovoCyte Flow Cytometers (ACEA, USA).

\section{Quantitative real-time PCR}

Total RNA was extracted using TRIzol (Takara, Japan) and was synthesized using PrimeScript RT Kit (Takara, Japan) following the manufacturer's instructions. qRT-PCR was performed by using SYBR Green (Tiangen) on an ABI7500 instrument. Relative gene expression was analyzed using $2^{-\Delta \Delta \mathrm{Ct}}$ method. For mRNA, $\beta$-Actin was used as the internal control, and for miRNA, U6 was used as the internal control. Primer sequences were listed in Table 1.

\section{Western blotting}

Total proteins were prepared from cell lines using RIPA
Lysis and Extraction Buffer (Thermo Fisher Scientific, USA), followed by separation with SDS-PAGE and probed with anti-YAP1 (\#14074, Cell Signaling Technology, USA), anti-TEAD1 (\#12292, Cell Signaling Technology, USA) or anti- $\beta$-Actin (sc-70319, SantaCruz, USA). All antibodies were 1:1,000 diluted.

\section{Dual-luciferase activity assay}

$400 \mathrm{bp}$ of the 3'-UTR region of YAP 1 or TEAD 1 containing the binding site of miRNA-497-5p was cloned into pMIR reporter plasmid, which was transfected into A549 and H1299 cells using Lipofectamine 2000 reagent (Thermo Fisher Scientific, China). 36 hours later, firefly and renilla luciferase activities were measured by the DualLuciferase Assay System (Promega, USA).

\section{Statistical analysis}

Statistical analysis was performed using GraphPad Prism 5. All experiments were conducted at least three times. All data were presented as mean \pm standard deviation (SD). Twotailed Student's $t$ test was used to calculate the statistical differences, with $\mathrm{P}$ value $<0.05$ was considered statistically different.

\section{Results}

\section{MiR-497-5p expression is downregulated in lung cancer tissues and cells}

To explore the expressional change of miR-497-5p in human NSCLC, we analyzed the level in tumor tissues and cells. qRT-PCR results demonstrated a significantly diminished expression of miR-497-5p in NSCLC tumors compared with normal non-cancerous tissues (Figure 1A). Our results are consistent with NSCLC patient data from Starbase website (Figures S1,S2). Moreover, the level of 
A

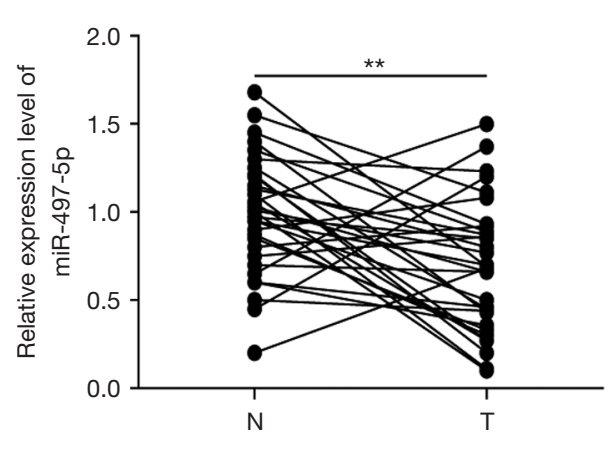

C

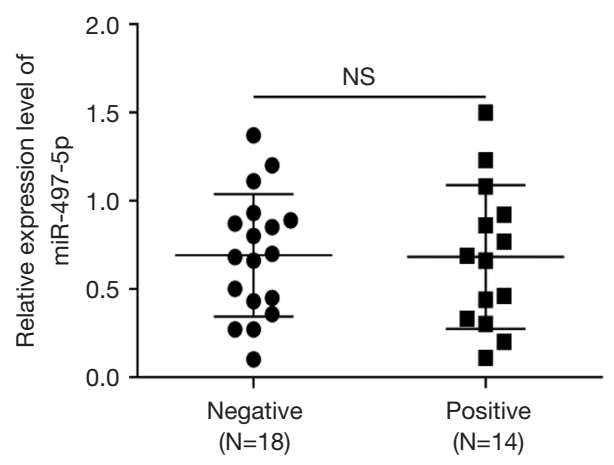

B

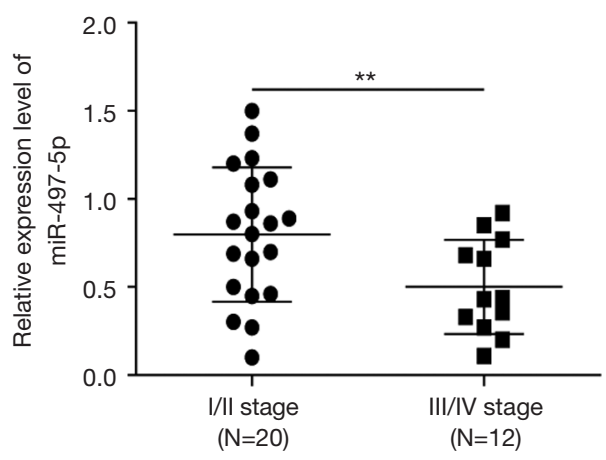

D

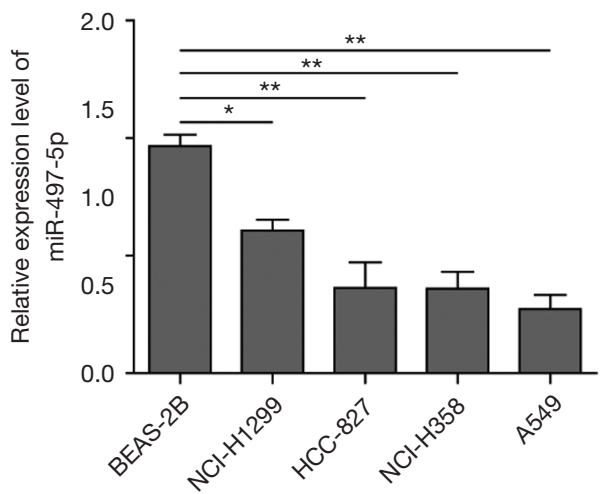

Figure 1 MiR-497-5p expression is downregulated in lung cancer tissues and cells. (A) The expression level of miR-497-5p was examined in lung cancer tissues and matched adjacent normal tissues by qRT-PCR. (B,C) The correlation between miR-497-5p expression and cancer stages (B) or lymph node metastasis (C) in NSCLC patients was analyzed. (D) The expression level of miR-497-5p was examined in human lung cancer cell lines and a non-cancerous lung epithelial cell line BEAS-2B by qRT-PCR. Data were mean \pm SD and were representative of three independent experiments. ${ }^{*} \mathrm{P}<0.05 ;{ }^{* *} \mathrm{P}<0.01$. NS, not significant.

miR-497-5p was negatively associated the cancer stages in NSCLC patients (Figure $1 B$ ). On the other hand, miR-497-5p expression did not correlate with lymph node metastasis (Figure 1C). Moreover, miR-497-5p expression was lower in lung cancer cell line A549, NCI-H358, HCC-827 and NCI-H1299 than that in non-cancerous lung epithelial cell line BEAS-2B (Figure 1D). Therefore, the development of lung cancer is associated with the decreased expression of miR-497-5p.

\section{MiR-497-5p triggers growth inbibition and apoptosis in lung cancer cells}

We first examined whether miR-497-5p could affect the proliferation of lung cancer cells. Results from CCK-8 experiment showed that in A549 and H1299 cells, overexpression of miR-497-5p significantly suppressed cell growth, which was increased by miR-497-5p inhibition (Figure 2A). Similarly, in a colony formation assay, the proliferation of A549 and H1299 cells was negatively regulated by miR-497-5p overexpression and was positively regulated by miR-497-5p inhibition (Figure $2 B$ ). Furthermore, overexpression of miR-497-5p led to significantly increased percentage of G0/G1-phase cells, whereas miR-497-5p inhibition caused the opposite effect, suggesting that miR-497-5p caused a G0/G1-phase cell cycle arrest (Figure 2C). On the other hand, overexpression of miR497-5p significantly increased the percentage of AnnexinVpositive apoptotic cells, whereas miR-497-5 inhibition reduced cell apoptosis (Figure 2D). Therefore, miR-497-5p 
A A549

H1299
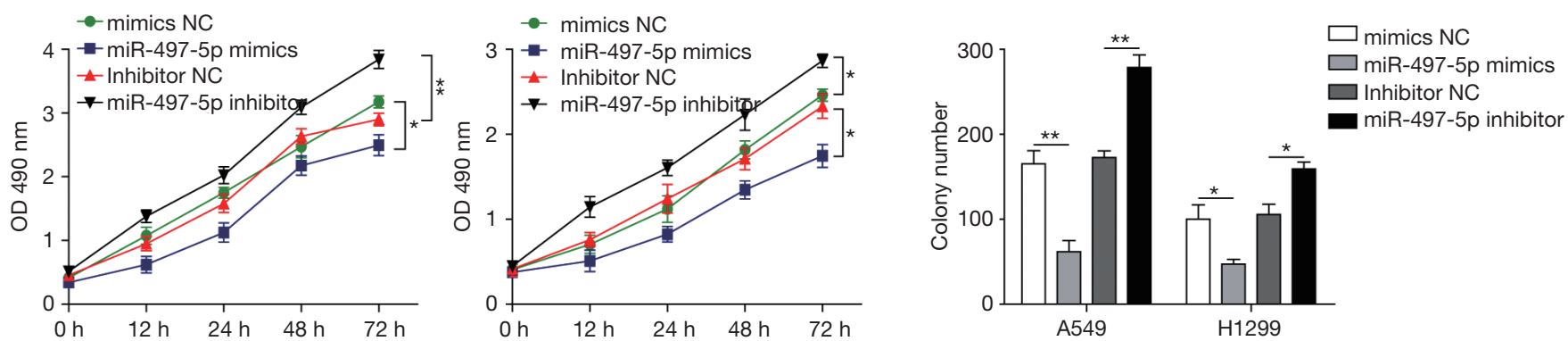

B
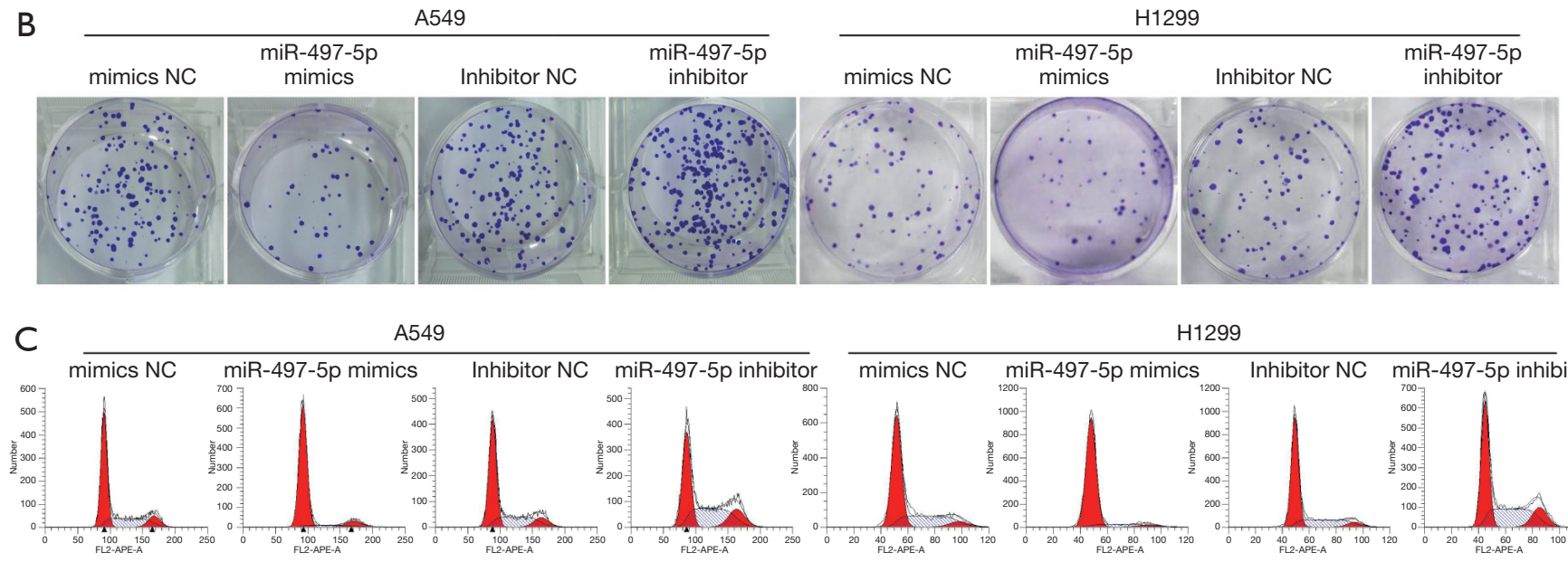

H1299
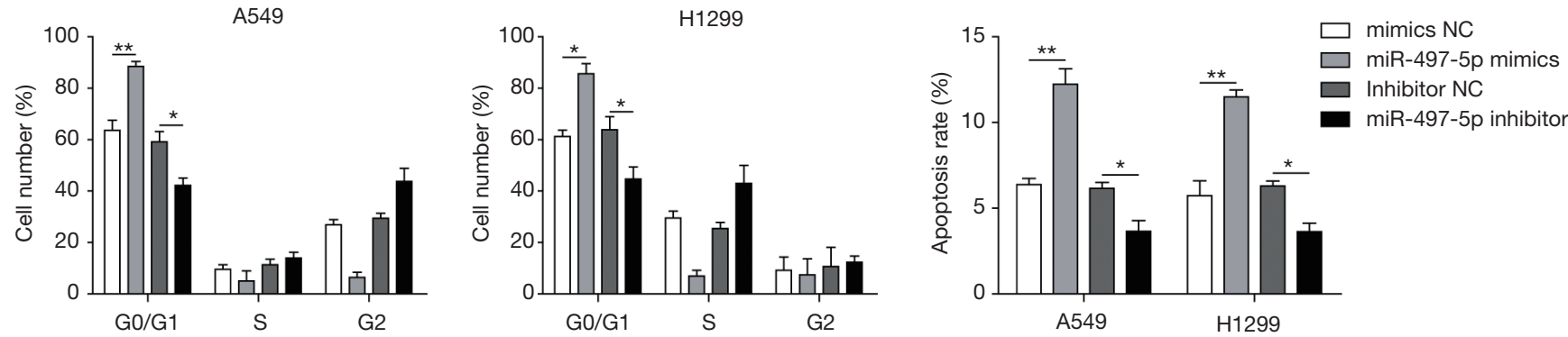

$\mathrm{D}$
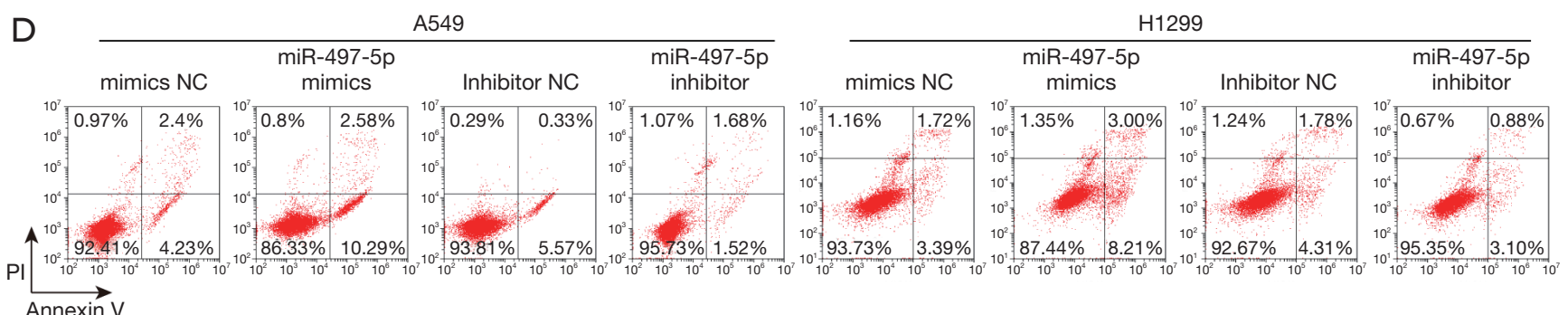

Figure 2 MiR-497-5p inhibits the proliferation and induces the apoptosis of lung cancer cells. A549 and H1299 cells were transfected with miR-497-5p mimic or miR-497-5p inhibitor, cell growth was evaluated by CCK-8 assay (A) and colony-formation assay (B). Cell cycle (C) and cell apoptosis (D) were evaluated by flow cytometry. Data were mean \pm SD and were representative of three independent experiments. ${ }^{*} \mathrm{P}<0.05$; ${ }^{* *} \mathrm{P}<0.01$. 
negatively regulates the growth of lung cancer cells.

\section{MiR-497-5p suppresses the expression of YAP1 and TEAD1}

Next, the potential target genes of miR-497-5 were predicted in TargetScan database in total online: http:// fp.amegroups.cn/cms/6aa 9 bbd 73 a $842 \mathrm{fd} 2$ a 8 bd 0 ba $16 \mathrm{e} 0$ e913f/tcr.2019.10.03-1.pdf. We found that the 3'-UTR region of YAP1 and TEAD1, two Hippo signaling effectors which were reported to be oncogenic, harbored the binding site of miR-497-5p (Figure $3 A$ ). To further validate the interaction between miR-497-5p and YAP1/TEAD1, A549 cells were transfected with miR-497-5 mimic or inhibitor. The expression levels of both YAP1 and TEAD1 were significantly decreased in miR-497-5p-overexpressed cells. Contrarily, miR-497-5p inhibition significantly increased the expression of YAP1 and TEAD1 (Figure 3B). The ability of miR-497-5p to downregulate YAP1 and TEAD1 was also supported by western blot at the protein level (Figure 3C). In a luciferase activity assay, miR-497-5p dampened the luciferase intensity in the lysates of A549 cells and H1299 expressing wild type 3'-UTR of YAP1 and TEAD1 but not in those expressing mutant 3 '-UTR of YAP1 and TEAD1 (Figure 3D). Furthermore, the abundance of both YAP1 and TEAD1 was increased in lung cancer tissues than those in paired normal lung tissues (Figure 3E). Importantly, their expression was negatively correlated with the level of miR497-5p (Figure 3F). Collectively, miR-497-5p suppresses the expression of YAP1 and TEAD1 in lung cancer cells.

\section{MiR-497-5p inbibits lung cancer cell proliferation by downregulating YAP1 and TEAD1}

We then explored whether miR-497-5p affect lung cancer cell function via modulating the expression of YAP1 and TEAD1. As shown in Figure 4A, the growth of A549 and H1299 cells was inhibited by miR-497-5p, but this growth retardation was reversed by the overexpression of YAP1 or TEAD1 (Figure 4A). Consistently, miR-497-5p mimic reduced the colony-forming ability in A549 and H1299 cells, but this effect of was largely abrogated by the concomitant overexpression of YAP 1 and TEAD 1 (Figure $4 B$ ). Moreover, forced expression of either YAP1 or TEAD1 largely reversed the G0/G1-phase cell cycle arrest and cell apoptosis induced by miR-497-5p overexpression (Figure $4 C, D$ ). As expected, the expression of YAP1 or TEAD1 was decreased by miR-497-5p and was rescued by YAP1 or TEAD1 overexpression in A549 cells (Figure 4E,F).
Therefore, miR-497-5p influences the proliferation and apoptosis via downregulating YAP1 and TEAD1.

\section{MiR-497-5p sensitizes lung cancer cells to cisplatin through the inbibition of YAP1 and TEAD1}

Interestingly, we found that the expression of miR-497-5p was markedly reduced in cisplatin-resistant A549/DDP cells than those in parental A549 cells (Figure 5A), and the expression of YAP1 and TEAD1 was increased in A549/ DDP cells (Figure $5 A, B$ ). These results prompted us to speculate that miR-497-5p may have a role in cisplatinresistance. Indeed, miR-497-5p overexpression significantly reduced the viability of A549/DDP cells upon cisplatin treatment as evidenced by CCK-8, and the IC50 values were significantly reduced in A549/DDP with miR-497-5p mimics when compared to NC groups (14.7 vs. $40.2 \mu \mathrm{M}$, Figure 5 C). By performing flow cytometry, we found that cisplatin treatment increased the apoptosis of A549 cells, and overexpression of miR-497-5p further sensitized cells to cisplatin treatment. This effect of miR-497-5p mimics was significantly abrogated by the overexpression of either YAP1 or TEAD1 (Figure 5D). In addition, cisplatin treatment downregulated the expression of both YAP1 or TEAD1, which was further decreased by miR-497-5p mimics and was rescued by YAP1 or TEAD1 overexpression in A549 cells (Figure 5E). Therefore, miR-497-5p enhances the chemosensitivity of lung cancer cells to cisplatin through targeting YAP1 and TEAD1.

\section{Discussion}

In recent years, increasingly more evidences have suggested that miRNAs play crucial role in regulating the proliferation, migration, invasion or drug resistance of NSCLC cells. Thus, identifying novel lung cancer-related miRNAs may be beneficial for the targeted intervention of NSCLC $(22,23)$.

As a downstream effector of Hippo signaling transduction pathway, YAP1 plays a crucial role in mediating organ development or the regeneration damaged organs (24). However, in the context of cancer, the oncogenic property of YAP1 was revealed by many previous works (25-27). In EGFR-dependent lung adenocarcinomas, the expression of YAP1 was increased and strongly supported the proliferation and survival of tumor cells (28). Pharmacological inactivation of YAP1 resulted in reduced growth and survival of lung cancer cells (28). Noto et al. has reported that YAP1 is indispensable for the maintenance of 
A

$\begin{array}{cc}\text { YAP1 3'UTR WT } & \text { CTCTTCCTTGTCCATTGCTGCTG } \\ \text { Hsa-miR-497-5p } & \text { UGUUUGGUGUCACACGACGAC } \\ \text { YAP13'UTR MUT } & \text { CTCTTCCTTGTCCATACGACGAG } \\ \text { TEAD1 3'UTR WT } & \text { CAGCCTCACCGGATGCTGCT } \\ \text { Hsa-miR-497-5p } & \text { UGUUUGGUGUCACACGACGAC } \\ \text { TEAD1 3'UTR MUT } & \text { CAGCCTCACCGGAACGACGA }\end{array}$

B



H1299

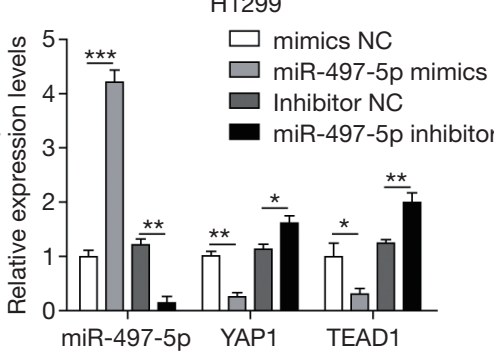

C
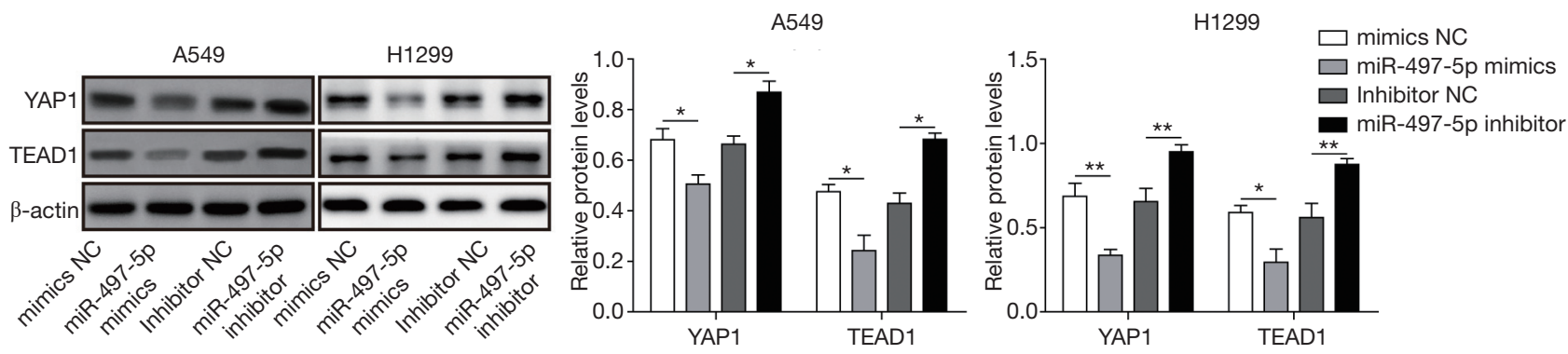

D
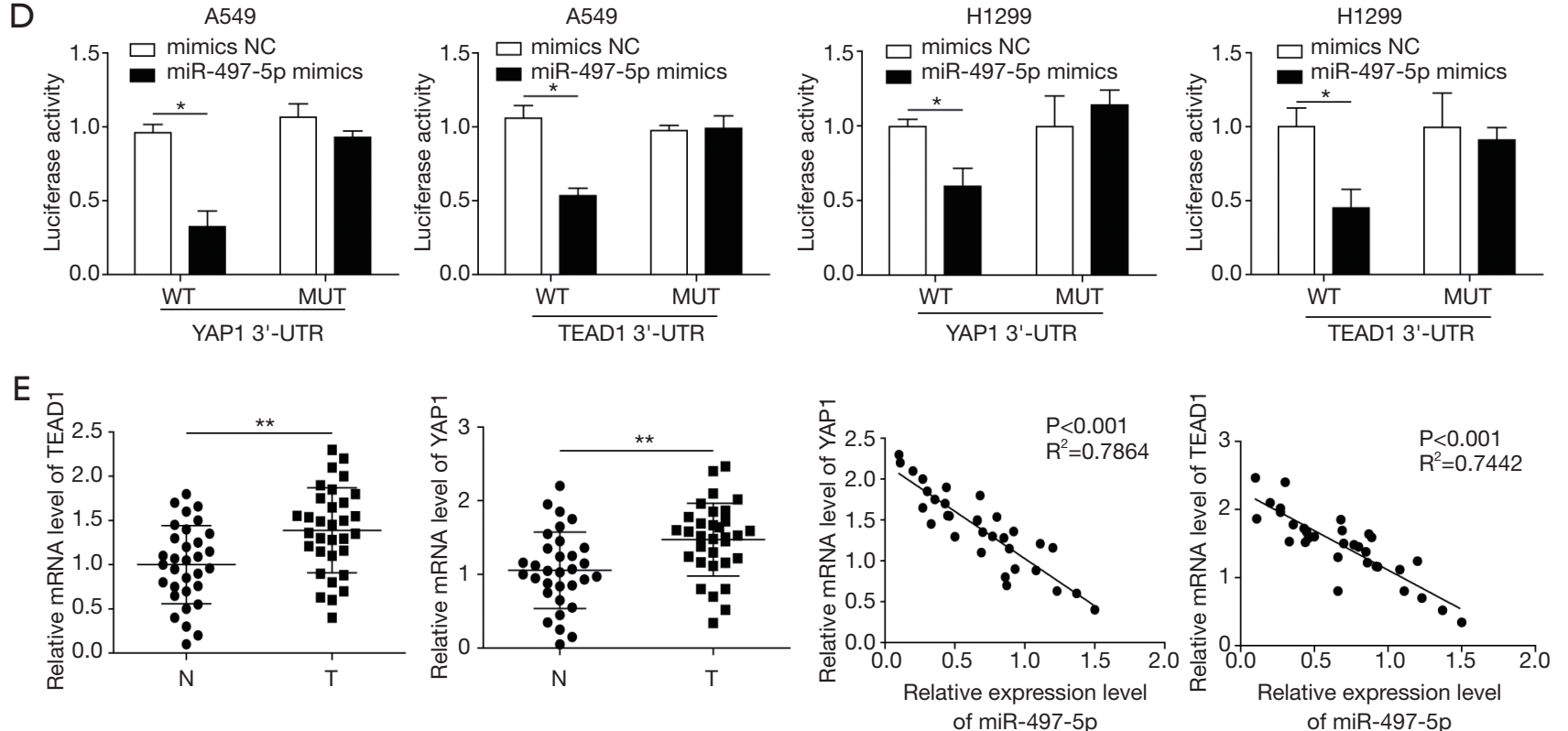

Figure 3 YAP1 and TEAD1 are targeted by miR-497-5p. (A) Sequence comparison between miR-497-5p and the 3'-UTR region of YAP1 and TEAD1. A549 cells were transfected with miR-497-5p mimic or miR-497-5p inhibitor, the levels of YAP1 and TEAD1 are detected by qRT-PCR (B) and Western blot (C). (D) A549 and H1299 cells were co-transfected with miR-497-5p mimic and luciferase vectors carrying 3'-UTR of YAP1 or TEAD1, the luciferase activity was measured. (E) The levels of YAP1 and TEAD1 in clinical cancer specimens were evaluated by qRT-PCR. (F) The correlation between miR-497-5p and YAP1/TEAD1 was evaluated by Spearman's rank correlation test. Data were mean $\pm \mathrm{SD}$ and were representative of three independent experiments. ${ }^{*} \mathrm{P}<0.05 ;{ }^{* *} \mathrm{P}<0.01$; ${ }^{* * *} \mathrm{P}<0.001$.

stemness of lung cancer cells, and high expression of YAP1 predicts poor prognosis in lung cancer patients (29).

TEAD transcription factor family is also crucial effectors in Hippo signaling cascade whose roles have been implicated in cell proliferation and differentiation. TEAD proteins constitute a highly conserved TEA DNA binding domain, a proline rich region and a YAP binding domain $(30,31)$. The abnormally upregulated expression of TEAD1 

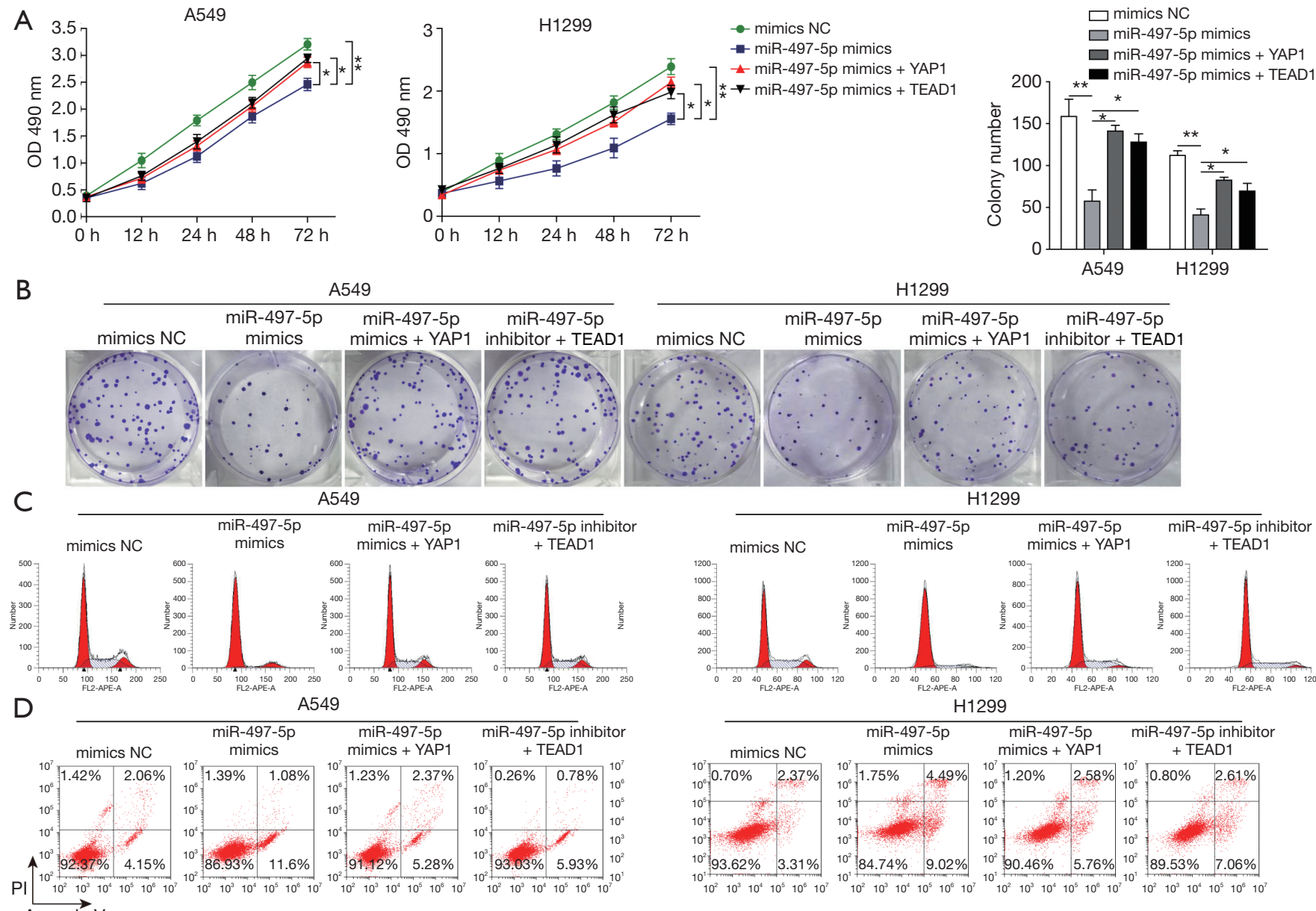

$\underset{\text { Annexin } \mathrm{V}}{\longrightarrow}$
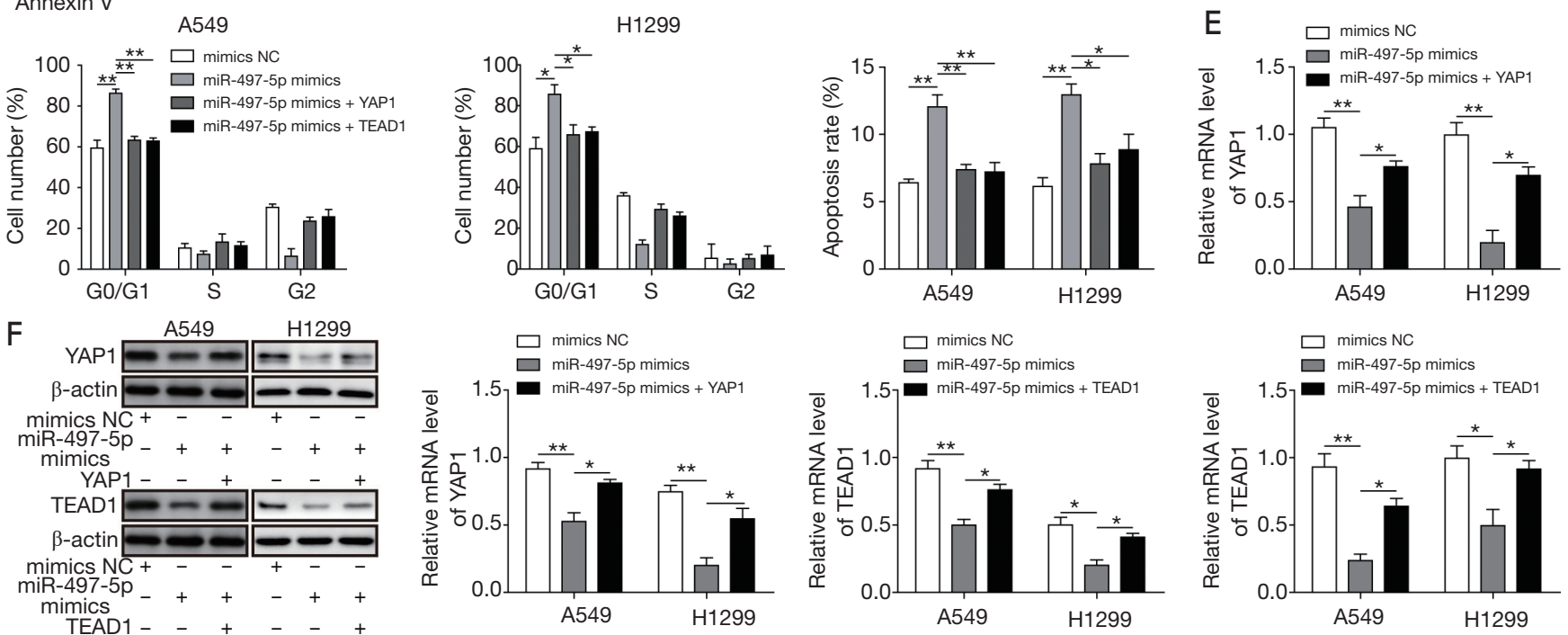

Figure 4 MiR-497-5p regulates the function of lung cancer cells via suppressing YAP1 and TEAD1. A549 and H1299 cells were cotransfected with miR-497-5p mimic and YAP1- or TEAD1-expressing plasmids, cell proliferation was measured by CCK-8 assay (A) and colony-formation assay (B). Cell cycle (C) and cell apoptosis (D) were evaluated by flow cytometry. The expression of YAP1 or TEAD1 was determined by qRT-PCR (E) and Western blot $(\mathrm{F})$. Data were mean \pm SD and were representative of three independent experiments. ${ }^{*} \mathrm{P}<0.05 ;{ }^{* *} \mathrm{P}<0.01$. 
A

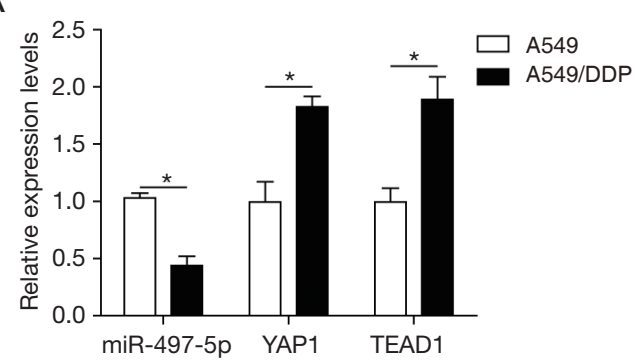

B
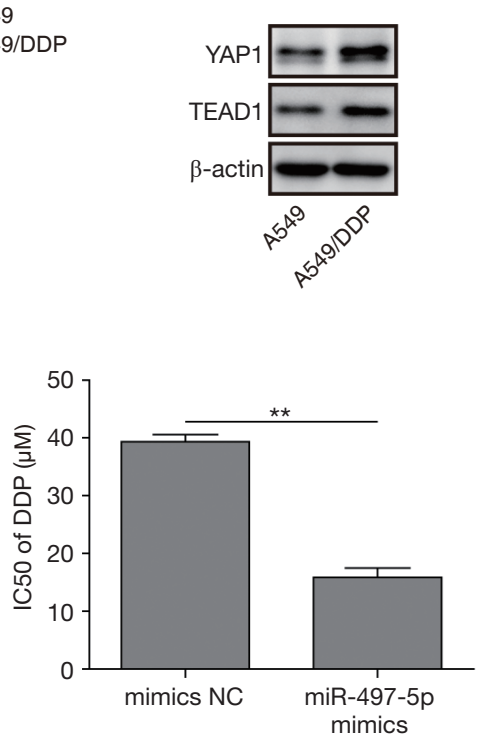
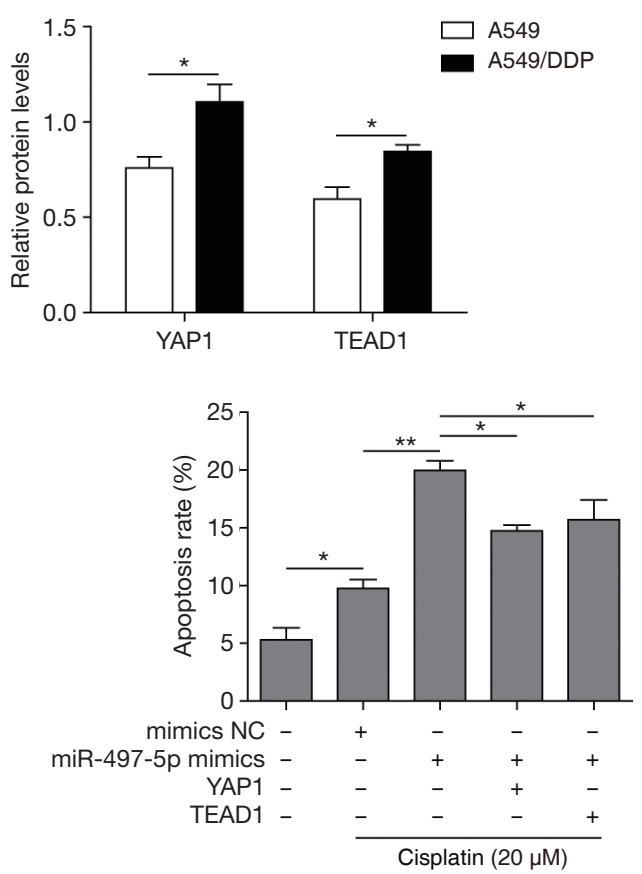

D

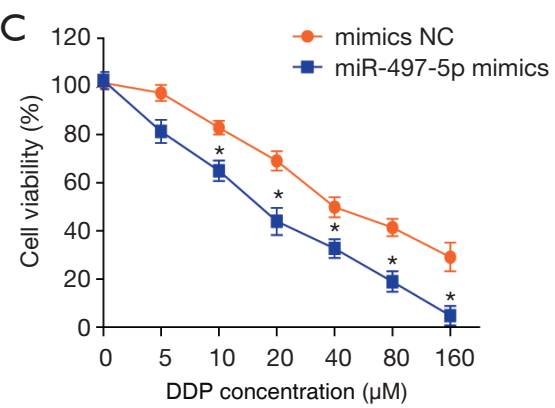

Cisplatin $(20 \mu \mathrm{M})$

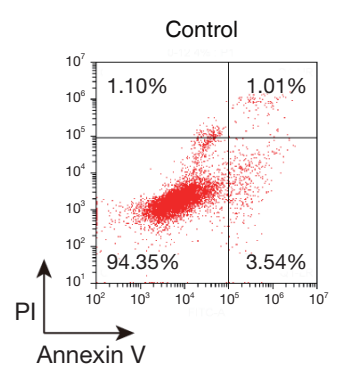

$\mathrm{E}$

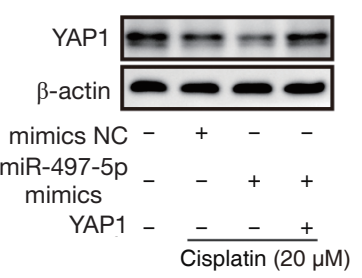

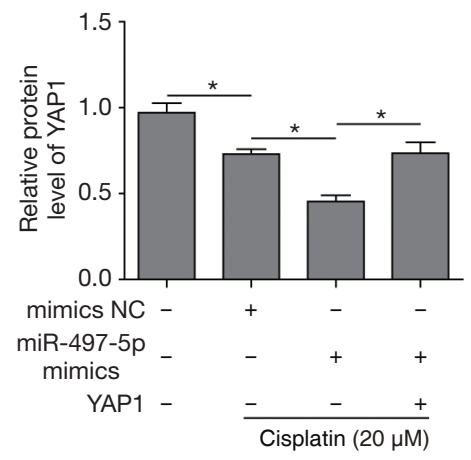
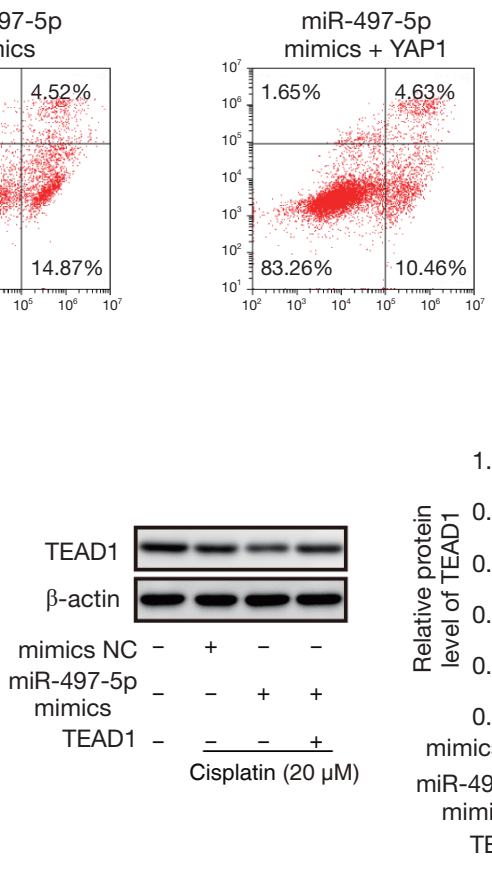

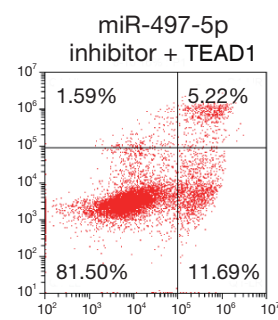

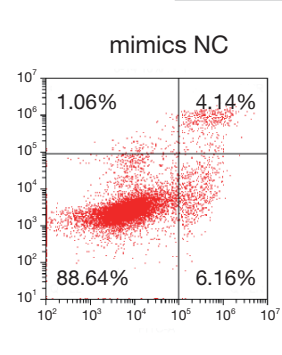
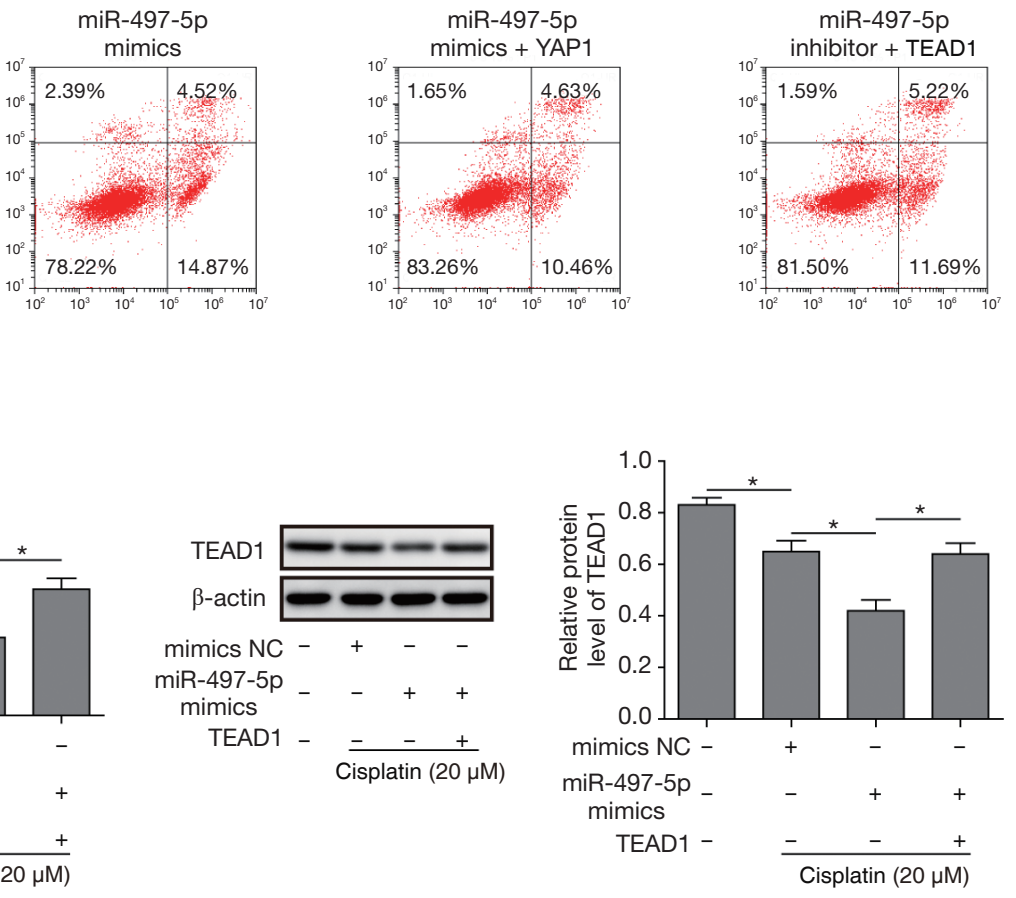

Figure 5 MiR-497-5p sensitizes lung cancer cells to cisplatin through the inhibition of YAP1 and TEAD1. (A) The expression miR-497-5p, YAP1 and TEAD1 in A549 cells and A549/DDP cells was evaluated by qRT-PCR. (B) The expression YAP1 and TEAD1 in A549 cells and A549/DDP cells was evaluated by western blot. (C,D) A549/DDP cells was transfected with miR-497-5p mimic followed by treatment with cisplatin, cell viability was evaluated by CCK-8 assay (C), cell apoptosis was measured by flow cytometry (D). (E) The levels of YAP1 and TEAD1 was evaluated in cisplatin-challenged A549/DDP cells by western blot. Data were mean \pm SD and were representative of three independent experiments. ${ }^{*} \mathrm{P}<0.05 ;{ }^{* *} \mathrm{P}<0.01$. 
is seen in many tumor types. In gastric carcinogenesis, TEAD1 is significantly increased in tumor tissues and promoted tumor cell malignancy. Zhou et al. have reported that TEAD1 was targeted and downregulated by miR-4269, whose expression was associated with good prognosis in gastric cancer patients, suggesting that miRNAs interfering TEAD1 expression is potentially tumor suppressive (32). In turn, YAP1 and TEAD1 could also induced the expression of several oncogenic microRNAs in NSCLC cell lines (33). In our present work, miR-497-5p was found to target the 3'-UTR of both YAP1 and TEAD1, demonstrating the suppressive role of miR-497-5p on Hippo signaling. The negative correlation between the level of miR-497-5p and YAP1 as well as TEAD1 in NSCLC patients indicates that the potential value of miR-497-5p in the clinical diagnosis or treatment of NSCLC. Cell function analysis showed that miR-497-5p inhibited the growth and promoted the apoptosis of A549 cells by targeting YAP1 and TEAD1.

To date, cisplatin resistance has emerged as a major problem in the treatment of NSCLC, which limited the clinical implication of cisplatin. It has been shown by several studies that miRNAs contribute to the cisplatin resistance in NSCLC. For example, miRNA-328 and miRNA-130b enhanced cisplatin resistance in NSCLC cells $(18,34)$. On the other hand, miR9 and miR-29c increased cisplatin sensitivity in NSCLC cells $(35,36)$. In our work, miR-497-5p is identified as a novel miRNA that disrupts the cisplatin resistance in lung cancer.

\section{Conclusions}

The present study demonstrated that miR-497-5p suppressed proliferation and cisplatin resistance of lung cancer cells through downregulating YAP1 and TEAD1, providing a novel candidate for the targeted intervention and therapeutics of NSCLC.

\section{Acknowledgments}

Funding: This study was supported by Jiangxi Provincial Natural Science Foundation of China (no. 20171BAB205058).

\section{Footnote}

Conflicts of Interest: The authors have completed the ICMJE uniform disclosure form (available at http://dx.doi. org/10.21037/tcr.2019.10.03). The authors have no conflicts of interest to declare.
Etbical Statement: The authors are accountable for all aspects of the work in ensuring that questions related to the accuracy or integrity of any part of the work are appropriately investigated and resolved. The study was conducted in accordance with the Declaration of Helsinki (as revised in 2013). The study has been approved by the Hospital's Ethics Committee (Approval Number: CDYFY2017-8999). Inform consent was obtained from all patients.

Open Access Statement: This is an Open Access article distributed in accordance with the Creative Commons Attribution-NonCommercial-NoDerivs 4.0 International License (CC BY-NC-ND 4.0), which permits the noncommercial replication and distribution of the article with the strict proviso that no changes or edits are made and the original work is properly cited (including links to both the formal publication through the relevant DOI and the license). See: https://creativecommons.org/licenses/by-nc-nd/4.0/.

\section{References}

1. Bray F, Ferlay J, Soerjomataram I, et. al. Global cancer statistics 2018: GLOBOCAN estimates of incidence and mortality worldwide for 36 cancers in 185 countries. CA Cancer J Clin 2018;68:394-424.

2. Ettinger DS, Aisner DL, Wood DE, et al. NCCN Guidelines Insights: Non-Small Cell Lung Cancer, Version 5. 2018. J Natl Compr Canc Netw 2018;16:807-21.

3. Asao T, Takahashi F, Takahashi K. Resistance to molecularly targeted therapy in non-small-cell lung cancer. Respir Investig 2019;57:20-6.

4. Yoda S, Dagogo-Jack I, Hata AN. Targeting oncogenic drivers in lung cancer: Recent progress, current challenges and future opportunities. Pharmacol Ther 2019;193:20-30.

5. Ardizzoni A, Boni L, Tiseo M, et al. Cisplatin- versus carboplatin-based chemotherapy in first-line treatment of advanced non-small-cell lung cancer: an individual patient data meta-analysis. J Natl Cancer Inst 2007;99:847-57.

6. Kim DW, Kim KC, Kim KB, et al. Transcriptional deregulation underlying the pathogenesis of small cell lung cancer. Transl Lung Cancer Res 2018;7:4-20.

7. Treiber T, Treiber N, Meister G. Regulation of microRNA biogenesis and its crosstalk with other cellular pathways. Nat Rev Mol Cell Biol 2019;20:5-20.

8. Rupaimoole R, Slack FJ. MicroRNA therapeutics: towards a new era for the management of cancer and other diseases. Nat Rev Drug Discov 2017;16:203-22. 
9. Inamura K, Ishikawa Y. MicroRNA In Lung Cancer: Novel Biomarkers and Potential Tools for Treatment. J Clin Med 2016;5. doi: 10.3390/jcm5030036.

10. Zheng W, Zhao J, Tao Y, et al. MicroRNA-21: A promising biomarker for the prognosis and diagnosis of non-small cell lung cancer. Oncol Lett 2018;16:2777-82.

11. Del Vescovo V, Denti MA. microRNA and Lung Cancer. Adv Exp Med Biol 2015;889:153-77.

12. Krutakova M, Sarlinova M, Matakova T, et al. The Role of Dysregulated MicroRNA Expression in Lung Cancer. Adv Exp Med Biol 2016;911:1-8.

13. Foss KM, Sima C, Ugolini D, et al. miR-1254 and miR574-5p: serum-based microRNA biomarkers for early-stage non-small cell lung cancer. J Thorac Oncol 2011;6:482-8.

14. Landi MT, Zhao Y, Rotunno M, et al. MicroRNA expression differentiates histology and predicts survival of lung cancer. Clin Cancer Res 2010;16:430-41.

15. Gasparini P, Cascione L, Landi L, et al. microRNA classifiers are powerful diagnostic/prognostic tools in ALK-, EGFR-, and KRAS-driven lung cancers. Proc Natl Acad Sci U S A 2015;112:14924-9.

16. Cui R, Meng W, Sun HL, et al. MicroRNA-224 promotes tumor progression in nonsmall cell lung cancer. Proc Natl Acad Sci U S A 2015;112:E4288-97.

17. Kang M, Shi J, Peng N, et. al. MicroRNA-211 promotes non-small-cell lung cancer proliferation and invasion by targeting MxA. Onco Targets Ther 2017;10:5667-75.

18. Wang C, Wang S, Ma F, et al. miRNA328 overexpression confers cisplatin resistance in nonsmall cell lung cancer via targeting of PTEN. Mol Med Rep 2018;18:4563-70.

19. Chen Y, Kuang D, Zhao X, et al. miR-497-5p inhibits cell proliferation and invasion by targeting $\mathrm{KCa} 3.1$ in angiosarcoma. Oncotarget 2016;7:58148-61.

20. Chai L, Kang XJ, Sun ZZ, et al. MiR-497-5p, miR-195$5 \mathrm{p}$ and $\mathrm{miR}-455-3 \mathrm{p}$ function as tumor suppressors by targeting hTERT in melanoma A375 cells. Cancer Manag Res 2018;10:989-1003.

21. Zhu D, Tu M, Zeng B, et al. Up-regulation of miR-497 confers resistance to temozolomide in human glioma cells by targeting mTOR/Bcl-2. Cancer Med 2017;6:452-62.

22. Lu J, Zhan Y, Feng J, et al. MicroRNAs associated with therapy of non-small cell lung cancer. Int J Biol Sci 2018;14:390-7.

23. Zaporozhchenko IA, Morozkin ES, Ponomaryova AA, et al. Profiling of 179 miRNA Expression in Blood Plasma of Lung Cancer Patients and Cancer-Free Individuals. Sci Rep 2018;8:6348.

24. Zhao B, Li L, Tumaneng K, et. al. A coordinated phosphorylation by Lats and CK1 regulates YAP stability through SCF (beta-TRCP). Genes Dev 2010;24:72-85.

25. Holden JK, Cunningham CN, et. al. Targeting the Hippo Pathway and Cancer through the TEAD Family of Transcription Factors. Cancers (Basel) 2018;10. doi: 10.3390/cancers 10030081.

26. Wu L, Yang X, et. al. Targeting the Hippo Pathway for Breast Cancer Therapy. Cancers (Basel) 2018;10. doi: 10.3390/cancers 10110422 .

27. Nakatani K, Maehama T, Nishio M, et al. Targeting the Hippo signalling pathway for cancer treatment. J Biochem 2017;161:237-44.

28. Lee TF, Tseng YC, Chang WC, et al. YAP1 is essential for tumor growth and is a potential therapeutic target for EGFR-dependent lung adenocarcinomas. Oncotarget 2017;8:89539-51.

29. Noto A, De Vitis C, Pisanu ME, et al. StearoylCoA-desaturase 1 regulates lung cancer stemness via stabilization and nuclear localization of YAP/TAZ. Oncogene 2017;36:4573-84.

30. Li Z, Zhao B, Wang P, et al. Structural insights into the YAP and TEAD complex. Genes Dev 2010;24:235-40.

31. Kaneko KJ, DePamphilis ML. Regulation of gene expression at the beginning of mammalian development and the TEAD family of transcription factors. Dev Genet 1998;22:43-55.

32. Zhou Y, Huang T, Zhang J, et al. TEAD1/4 exerts oncogenic role and is negatively regulated by miR-4269 in gastric tumorigenesis. Oncogene 2017;36:6518-30.

33. Lo Sardo F, Forcato M, Sacconi A, et al. MCM7 and its hosted miR-25, 93 and 106b cluster elicit YAP/TAZ oncogenic activity in lung cancer. Carcinogenesis 2017;38:64-75.

34. Zhang Q, Zhang B, Sun L, et al. MicroRNA-130b targets PTEN to induce resistance to cisplatin in lung cancer cells by activating Wnt/beta-catenin pathway. Cell Biochem Funct 2018;36:194-202.

35. Sun DM, Tang BF, Li ZX, et al. MiR-29c reduces the cisplatin resistance of non-small cell lung cancer cells by negatively regulating the PI3K/Akt pathway. Sci Rep 2018;8:8007.

36. Pan Q, Sun L, Zheng D, et al. MicroRNA-9 Enhanced Cisplatin Sensitivity in Nonsmall Cell Lung Cancer Cells by Regulating Eukaryotic Translation Initiation Factor 5A2. Biomed Res Int 2018;2018:1769040.

Cite this article as: Zeng SG, Xie JH, Zeng QY, Dai SH, Wang Y, Wan XM, Zhou XL. MicroRNA-497-5p negatively regulates the proliferation and cisplatin resistance of non-small cell lung cancer cells by targeting YAP1 and TEAD1. Transl Cancer Res 2019;8(6):2470-2480. doi: 10.21037/tcr.2019.10.03 


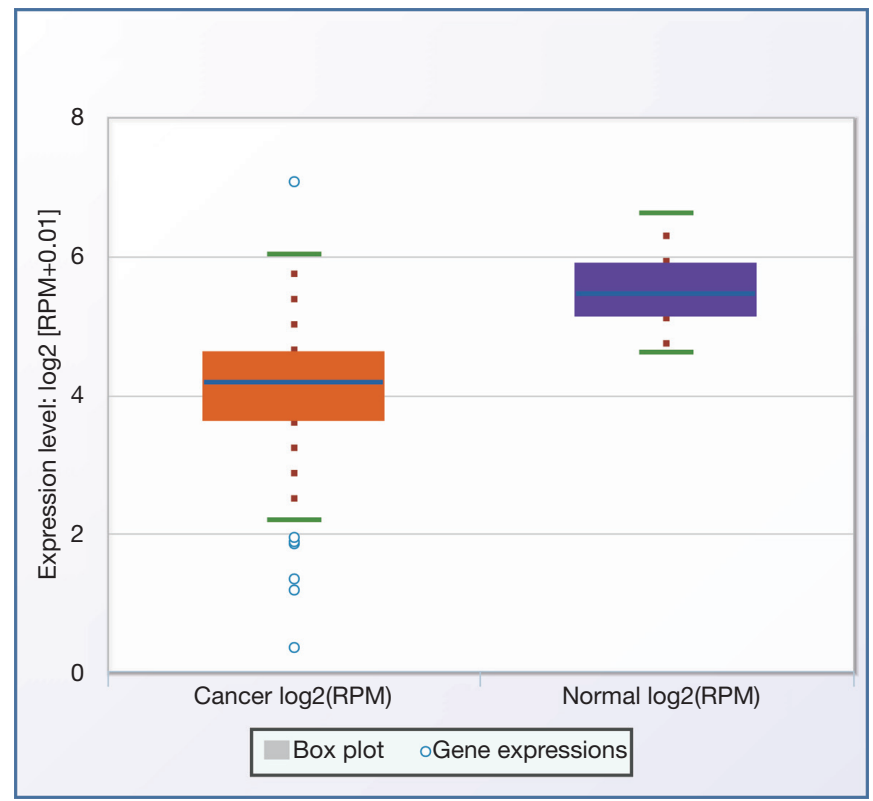

Figure S1 Has-miR-497-5p with 475 cancer and 38 normal samples in LUSC. Data source: starBase v3.0 project.

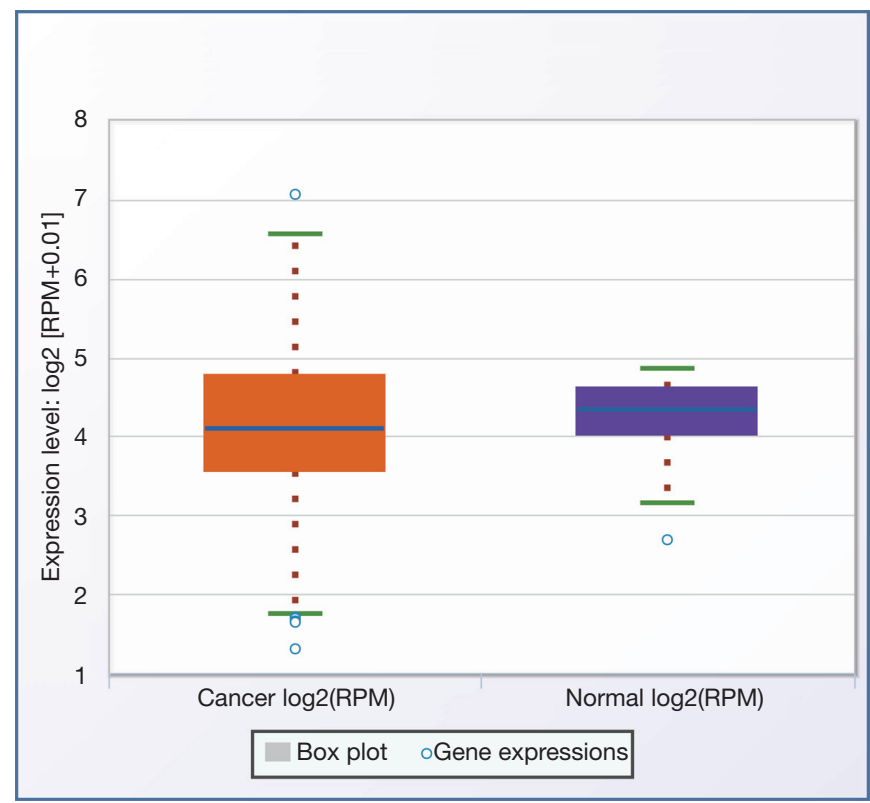

Figure S2 Has-miR-497-5p with 512 cancer and 20 normal samples in LUAD. Data source: starBase v3.0 project. 